\title{
Obesidade materna: Antropometria dos recém-nascidos
}

\section{Maternal obesity: Newborns anthropometry}

DOI: $10.46814 /$ lajdv4n1-002

Recebimento dos originais: 01/12/2021

Aceitação para publicação: 17/01/2022

\section{Prof. Dr. Joe Luiz Vieira Garcia Novo}

Professor Titular do Departamento de Reprodução Humana e Infância - Faculdade de Ciências Médicas e da Saúde - PUC/SP.

Rua Manoel Ferreira Leão, 67 ap 21 Vila Leão - Sorocaba-SP CEP 18040-410

E-mail: joeluiznovo@ hotmail.com

\section{Prof. Dr. Neil Ferreira Novo}

Ex - Professor Titular do Departamento de Morfologia - Faculdade de Ciências Médicas e da Saúde PUC/SP.

\author{
Alessandra Raphael Novelli \\ Ex - Acadêmica do Curso de Medicina - Faculdade de Ciências Médicas e da Saúde - PUC/SP. \\ Mariana Oliva Cassará Carvalho \\ Ex - Acadêmica do Curso de Medicina - Faculdade de Ciências Médicas e da Saúde - PUC/SP.
}

\section{RESUMO}

Objetivo: analisar valores antropométricos em recém-nascidos de parturientes obesas, assistidas na maternidade do Hospital Santa Lucinda de Sorocaba-SP. Métodos: estudo prospectivo com 200 parturientes: 100 obesas e 100 controle (não obesas). Como variável materna dependente considerouse a obesidade, as de controle foram: idade, cor, ganho ponderal, paridade, partos (vaginais e cesáreas) e evolução puerperal. Nos recém-nascidos as variáveis dependentes foram as medidas antropométricas (peso, comprimento, perímetros cefálico e braquial, IMC), e as de controle: vitalidade, gênero e evolução no berçário. Resultados: o ganho médio de peso materno foi significantemente menor nas obesas $(15,2 \%)$ em relação ao grupo controle $(23,08 \%)(p=0,0001)$. Em 47,0\% das obesas os partos se resolveram através de cesáreas e $29,0 \%$ no grupo controle $(\mathrm{p}=0,01)$. As médias das medidas dos recém-nascidos: peso: 3,35 e 3,18k $(\mathrm{p}=0,005)$, perímetro cefálico: 34,3 e 33,6 cm $(\mathrm{p}=0,002)$, comprimento: 49,5 e 49,0 cm ( $\mathrm{p}=0,05)$, perímetro braquial esquerdo médio: 10,7 e 10, $3 \mathrm{~cm}(\mathrm{p}=0$, 001), IMC: 13,6 e 13,44 kg/m2 ( $\mathrm{p}=0,24)$ nos grupos de estudo e controle respectivamente. Conclusões: nesta amostra de mulheres obesas houve significativa e estatisticamente maiores proporções de partos cirúrgicos, além de recém-nascidos com valores antropométricos superiores (peso, perímetros cefálico e braquial e comprimento) em relação aos das pacientes não obesas. Mulheres obesas em idade reprodutiva, devem ser informadas dos prováveis riscos maternos e/ou fetais antes de uma futura gestação.

Palavras- chaves: obesidade materna, partos, antropometria, recém-nascidos.

\section{ABSTRACT}

Objective: to analyze anthropometric values in newborns of obese parturients, assisted in the maternity hospital of Santa Lucinda de Sorocaba-SP. Methods: prospective study with 200 parturients: 100 obese 
and 100 control (non-obese). Obesity was considered the dependent maternal variable, the control ones being age, color, weight gain, parity, deliveries (vaginal and cesarean) and puerperal evolution. In newborns, the dependent variables were anthropometric measurements (weight, length, head and brachial perimeters, BMI), and the control variables: vitality, gender and evolution in the nursery. Results: the mean maternal weight gain was significantly lower in the obese (15.2\%) compared to the control group $(23.08 \%)(\mathrm{p}=0.0001)$. In $47.0 \%$ of obese women, deliveries were resolved through cesarean sections and $29.0 \%$ in the control group $(\mathrm{p}=0.01)$. Means of newborns' measurements: weight: 3.35 and $3.18 \mathrm{k}(\mathrm{p}=0.005)$, head circumference: 34.3 and $33.6 \mathrm{~cm}(\mathrm{p}=0.002)$, length: 49.5 and $49.0 \mathrm{~cm}(\mathrm{p}=0.05)$, mean left arm circumference: 10.7 and $10.3 \mathrm{~cm}(\mathrm{p}=0.001), \mathrm{BMI}: 13.6$ and $13.44 \mathrm{~kg} / \mathrm{m} 2(\mathrm{p}=0,24)$ in the study and control groups respectively. Conclusions: in this sample of obese women, there were significantly and statistically higher proportions of surgical deliveries, in addition to newborns with higher anthropometric values (weight, head and arm circumferences and length) compared to non-obese patients. Obese women of childbearing age should be informed of the likely maternal and/or fetal risks before a future pregnancy.

keyword: maternal obesity, births, anthropometry, newborns.

\section{INTRODUÇÃO}

A fecundação e a implantação do ovo criam no organismo feminino modificações gerais e locais, favorecendo o relacionamento metabólico harmônico com o conjunto embrionário. O desenvolvimento embrionário e fetal está diretamente associado às ofertas de oxigênio e nutrientes, é influenciado por fatores genéticos que são mais ativos no início da gravidez, porém, sob estreita interligação materna, perduram até o término do período gestacional. ${ }^{1-4}$

Durante a gravidez existem duas fases metabólicas distintas. Na fase inicial (anabolismo materno-fetal) a mãe desenvolve retenção gordurosa em seu tecido adiposo, queimando parte da glicose ingerida utilizando-a para seus gastos energéticos, e concomitantemente gerenciando suporte energético ao conteúdo embrionário em desenvolvimento. ${ }^{5}$

$\mathrm{Na}$ fase seguinte (catabolismo materno e anabolismo fetal), a partir do terceiro trimestre gestacional, a fonte energética do organismo materno provém da queima de seus depósitos lipídicos, promovendo e elevando a passagem de glicose materna, para que haja o perfeito desenvolvimento do feto. 6

Dentro desta fisiológica intimidade materno-fetal, porém, a presença de patologias maternas clínicas e/ou obstétricas preexistentes, ou que se desenvolvam exclusivamente durante a gestação, no parto e/ou no puerpério, poderão desequilibrar esta harmonia inicial. Entre as patologias clínicas preexistentes, temos a obesidade materna pré-conceptual. ${ }^{7}$

A síndrome da obesidade apresenta-se como um distúrbio ou alteração da composição corporal, demonstrada pela presença de excesso absoluto e/ou relativo de gordura corporal. Em outras palavras é uma condição caracterizada, pelo acúmulo excessivo de gordura em um determinado organismo. ${ }^{7}$ 
$\mathrm{Na}$ atualidade entende-se que a obesidade represente patologia de múltiplas causas, acometendo pessoas com susceptibilidade de natureza genética, e submetida a diversas influências ambientais. Favorece-se um balanço energético e metabólico positivo além de ganho de peso. Nas três últimas décadas a prevalência da obesidade vem aumentando de maneira significativa, constante e crescente na população adulta e mesmo, na infantil. ${ }^{7,8}$

Em relação ao ciclo grávido puerperal na opinião de vários autores, a obesidade materna está associada a prováveis complicações ao binômio materno-fetal, relacionadas aos tipos de partos e/ou outras, decorrentes do aumento do peso do futuro recém-nascido ${ }^{5-7}$ e, portanto, de suas características antropométricas. ${ }^{5,8-12}$

A avaliação a respeito destas prováveis complicações decorrentes da obesidade materna, coexistentes durante o ciclo grávido puerperal, demonstra que ainda pairam dúvidas a respeito do modelo assistencial, a estas pacientes e a seus recém-nascidos. ${ }^{8,13}$

Nesta pesquisa propôs-se a analisar as prováveis repercussões da síndrome de obesidade materna em relação aos valores antropométricos de seus recém-nascidos, através de partos em maternidade de assistência obstétrica secundária.

\section{MÉTODOS}

Realizou-se estudo prospectivo de parturientes obesas e seus recém-nascidos, assistidos na maternidade e berçário do Hospital Santa Lucinda de Sorocaba - SP (HSL). Analisaram-se 200 pacientes e seus recém-natos, sendo 100 do grupo de desenho (pacientes obesas), e 100 (não obesas) em grupo de estudo comparativo, que concordaram e assinaram afirmativamente o Termo de Consentimento Livre e Esclarecido para esta pesquisa.

Os critérios de inclusão das pacientes do grupo de estudo antes da parturição, nesta pesquisa foram: o acompanhamento pré-natal iniciou-se durante o primeiro trimestre da gravidez; confirmação de feto único e sem malformações, em desenvolvimento, no mínimo, com 37 semanas completas e que a patologia materna associada à gravidez seria somente obesidade na consulta inicial da assistência pré-natal.

Para a definição nutricional de gestantes na atualidade, utilizaram-se medidas e índices antropométricos. A tendência mais utilizada foi acompanhar as orientações da Organização Mundial de Saúde (OMS), que recomenda neste enfoque reconhecer o Índice de Massa Corporal (IMC) préconceptual como procedimento adequado. ${ }^{13}$

A obesidade materna foi avaliada através do IMC que é calculado dividindo-se o peso corporal (em quilogramas) pelo quadrado da altura corporal (em metros). A classificação do peso materno foi conceituada como: baixo peso (IMC < 18,5), peso normal (IMC= 18,5 - 24,9), obesidade classe I ou 
sobrepeso $(\mathrm{IMC}=25,0-29,9)$, obesidade classe II $(\mathrm{IMC}=30,0-39,9)$ e obesidade extrema ou classe III (IMC $\geq 40,0){ }^{7}$

As pacientes consideradas como obesas (variável materna dependente) foram identificadas através de entrevista e carteira de pré-natal, conhecendo-se sua estatura em metros, peso em quilogramas e calculando-se o IMC do início da gravidez.. Anotaram-se como variáveis de controle: idade, cor, ganho ponderal, paridade, tipos de partos (vaginais e cesáreas), evolução puerperal e condições de alta materna.

Nos recém-nascidos foram estudados como variáveis dependentes: parâmetros antropométricos e IMC. Os parâmetros antropométricos foram representados pelo peso, comprimento, perímetros cefálico e braquial esquerdo; vitalidade, gênero e evolução no berçário foram as variáveis de controle. 14

O peso dos recém-natos foi obtido logo após o parto, eles sem roupas, em decúbito dorsal, utilizando-se balança elétrica marca WELMY previamente calibrada (padrões do Instituto Nacional de Pesos e Medidas), com capacidade máxima de peso de 15k, e a mínima de 125g, com subdivisões de 5g. ${ }^{5} \mathrm{O}$ peso foi anotado em gramas, e a adequação ponderal, analisada em tabela padronizada. ${ }^{15,16}$

O comprimento foi aferido em superfície rígida com régua antropométrica de madeira (divisões de 0,1 centímetros). A medida do perímetro cefálico foi realizada dentro das primeiras 24 horas de vida do recém-nascido em decúbito dorsal, com fita métrica não distensível, largura de $1 \mathrm{~cm}$ e subdivisões de $0,1 \mathrm{~cm} .^{5,17}$

O perímetro braquial foi avaliado com o mesmo tipo de fita, no membro superior esquerdo (E) do recém-nato em decúbito dorsal, com o braço em posição lateral ao tronco, cuidando-se para não enrugar a pele. O ponto médio foi medido entre as distâncias das extremidades do acrômio e olécrano, com o cotovelo fletido em ângulo de $90^{\circ} .16,17$

O cálculo pós-natal dos parâmetros físicos e neurológicos, estimou a idade gestacional dos recém-natos. ${ }^{18} \mathrm{O}$ IMC fetal foi calculado pela fórmula tradicional, e os seus valores de distribuição, acompanharam as tabelas conhecidas. ${ }^{5}$ Dados padronizados do exame físico dos recém-nascidos estimaram a qualidade das suas vitalidades. ${ }^{19}$

Os resultados obtidos por meio da avaliação do grupo de estudo, foram comparados aos do grupo de controle não obesas, isto é, com parturientes apresentando IMC dentro da faixa de normalidade atendidas na maternidade, apresentando as mesmas características das pacientes do grupo de estudo, exceto obesidade, durante o mesmo período da pesquisa. ${ }^{5}$

Os dados obtidos na consulta de prontuários, foram protegidos por Termo de Compromisso e Confidencialidade assinados pelos membros deste estudo. A pesquisa respeitou as normas da Declaração de Helsinque, ${ }^{20}$ e, da resolução no 466/2012 do Conselho Nacional de Saúde, para pesquisa 
em seres humanos. ${ }^{21}$ Os trabalhos iniciaram-se após a sua aprovação, através do Conselho de Ética em Pesquisa da Faculdade de Ciências Médicas e da Saúde-PUC/SP.

As pacientes incluídas foram puérperas de, no mínimo, seis horas de pós-parto, quando, em geral, elas já estavam descansadas, e, em condições de responder convictamente à entrevista consentida e informada. ${ }^{22}$

$\mathrm{Na}$ análise estatística em todos os testes fixou-se o nível de significância em 0,05 ou 5\%. Para a análise dos resultados foram aplicados os seguintes testes estatísticos:

1- Teste do quiquadrado ou teste exato de Fisher, com a finalidade de comparar os grupos de estudo e controle em relação à faixa etária, paridade, via de parto, índice de vitalidade de um minuto após o parto e gênero do recém-nascido. ${ }^{23}$

2- Teste t de Student para comparar os dois grupos acima quanto ao peso, perímetro braquial, comprimento, perímetro cefálico e IMC dos recém-nascidos. ${ }^{24}$

3- Teste de Mann-Whitney para comparar os dois grupos em relação ao aumento percentual ( $\Delta \%)$ do peso. Para o cálculo do $\Delta \%$ utilizou-se a fórmula: ${ }^{25}$

$$
\Delta \%=\frac{\text { peso final }- \text { peso inicial }}{\text { peso inicial }} \times 100
$$

\section{RESULTADOS}

Em ambos os grupos as variáveis maternas (idade, cor, paridade, puerpério e alta) e dos recémnatos (gênero, vitalidade e alta) foi satisfatória, não apresentaram diferenças estatisticamente significantes dispensando, portanto, tabelas demonstrativas.

O ganho médio de peso materno foi significantemente menor no grupo de estudo $(15,2 \%)$ em relação ao grupo de controle $(23,08 \%)$, isto é, z cálc. $=6,88(\mathrm{p}=0,001$. Observou-se diferença em relação à via de parto: no grupo de estudo: 47,0\% deles se resolveram através de cesárea, o que ocorreu em 29,0\% no grupo controle ( $\mathrm{p}=0,01)$ (Tabela 2). A média do peso de nascimento dos recém-nascidos foi de $3,35 \mathrm{~kg}$ no grupo de estudo e de $3,18 \mathrm{~kg}(\mathrm{p}=0,005)$ para o grupo controle (Tabela 3 ).

Tabela 1. Distribuição das pacientes segundo ganho percentual de peso.

\begin{tabular}{lll}
\hline GANHO \% DE PESO & CONTROLE & ESTUDO \\
\hline MÉDIA & $23,08 \%$ & $15,2 \%$ \\
MEDIANA & $21,5 \%$ & $14,3 \%$ \\
\hline
\end{tabular}

$\mathrm{z}$ calc $=6,11(\mathrm{p}<0,0001)$ 
Tabela 2. Distribuição das pacientes segundo a via de parto.

\begin{tabular}{lllll}
\hline \multirow{2}{*}{ VIA DE PARTO } & \multicolumn{2}{l}{ CONTROLE } & \multicolumn{2}{l}{ ESTUDO } \\
\cline { 2 - 6 } \cline { 3 - 5 } $\mathrm{N}^{\circ}$ & $\%$ & & \multicolumn{2}{l}{$\%$} \\
\hline Vaginal & 71 & $71,0 \%$ & 53 & $53,0 \%$ \\
Cirúrgica & 29 & $29,0 \%$ & 47 & $47,0 \%$ \\
\hline TOTAL & 100 & $100,0 \%$ & 100 & $100,0 \%$ \\
\hline
\end{tabular}

$\mathrm{x}^{2}$ calc $=6,88(\mathrm{p}=0,01)$

Tabela 3. Distribuição dos recém-nascidos segundo média do peso de nascimento

\begin{tabular}{lll}
\hline PESO RN (kg) & CONTROLE & ESTUDO \\
\hline MÉDIA & 3,18 & 3,35 \\
DP & 0,42 & 0,45 \\
\hline t calc $=2,65(\mathrm{p}=0,005)$ & &
\end{tabular}

As médias das medidas dos recém-nascidos foram: perímetro cefálico $34,3 \mathrm{~cm}$ e $33,6 \mathrm{~cm}(\mathrm{p}=$ 0,002), perímetro braquial 10,7 cm e 10,3 cm (p=0,001) e comprimento 49,5 cm e $49 \mathrm{~cm}(\mathrm{p}=0,05)$ respectivamente no grupo de estudo e de controle (Tabela 4). A média do IMC dos recém-natos foi de $13,63 \mathrm{~kg} / \mathrm{m}^{2}$ para o grupo de estudo e de $13,44 \mathrm{~kg} / \mathrm{m}^{2}$ para o grupo controle $(\mathrm{p}=0,24)$ (Tabela 5).

Tabela 4. Distribuição dos recém-nascidos segundo as mensurações.

\begin{tabular}{lllllll}
\hline & \multicolumn{2}{l}{ Perímetro cefálico $(\mathrm{cm})$} & \multicolumn{2}{l}{ Perímetro braquial E $(\mathrm{cm})$} & \multicolumn{2}{l}{ Comprimento $(\mathrm{cm})$} \\
\hline & Controle & Estudo & Controle & Estudo & Controle & Estudo \\
Média & 33,6 & 34,3 & 10,3 & 10,7 & 49 & 49,5 \\
DP & 1,5 & 1,8 & 0,9 & 1,0 & 1,9 & 2,0 \\
\hline & $\mathrm{t}$ calc $=2,94(\mathrm{p}=0,002)$ & t calc $=3,18(\mathrm{p}=0,001)$ & $\mathrm{t}$ calc $=1,65(\mathrm{p}=0,050)$
\end{tabular}

Tabela 5. Distribuição dos recém-nascidos segundo média do IMC.

\begin{tabular}{lll}
\hline IMC RN $(\mathrm{kg} / \mathrm{m} 2)$ & Controle & Estudo \\
\hline Média & 13,44 & 13,63 \\
DP & 2,4 & 1,3 \\
\hline $\mathrm{t}$ calc $=0,71(\mathrm{p}=0,24)$ & &
\end{tabular}

\section{DISCUSSÃO}

A obesidade cuja prevalência vem se elevando atualmente, representa grave problema de saúde pública. A gravidez pode atuar como fator desencadeante e/ou agravante, quando a obesidade for preexistente à gestação. Em consequência, há unanimidade entre os autores, a citação de que poderão se elevar os índices de morbiletalidade materna e fetal durante o ciclo grávido puerperal. ${ }^{8,10,27}$

Observou-se neste estudo, menor ganho ponderal materno nas obesas em desacordo com outros estudos. ${ }^{8,26}$ Os recém-nascidos de mães obesas apresentaram parâmetros antropométricos e características volumétricas estatisticamente superiores, e significantes comparados aos das pacientes não obesas, o que de certa maneira podem ter contribuído para que a resolução da via de parto através de cesáreas nas obesas, fosse mais elevada em relação ao grupo controle. ${ }^{10,12}$ 
Das repercussões da obesidade materna sobre o concepto, a macrossomia vem à vanguarda, podendo causar partos acidentados e outras consequências aos nascituros. ${ }^{10,12}$ Estes dados vêm corroborar citações em literaturas, pertinentes aos riscos da associação entre estado nutricional prégestacional, e a predisposição do risco de intercorrências gestacionais e de resolução do parto. $5,8,10,27$, $28-20$

As estatísticas de morbiletalidade materna e perinatal em países em desenvolvimento continuam fortemente preocupantes, uma vez de estão intimamente interligados ao não cumprimento de técnicas, cuidados maternos e perinatais de boa qualidade no transcorrer do ciclo grávido puerperal. $28-30$

Sinale-se que a possibilidade de beneficiar o estado de nutrição, e a qualidade de vida em longo prazo de um recém-nascido, limita-se a partir da concepção, a até os três primeiros anos de vida. ${ }^{29}$

Compete prioritariamente à saúde pública, identificar precocemente os fatores determinantes do comprimento ao nascer, conjuntamente com os do peso do recém-nato, para que possam gerar intervenções e condutas preventivas adequadas, criando-se consequentemente profilaxia para futuros danos maternos e/ou fetais, ${ }^{28}$ restabelecendo o respeito e à tão desejada autonomia da mulher. ${ }^{29}$

Mulheres com obesidade em idade reprodutiva devem ser informadas de seus riscos, associados a uma futura gestação, e atendidas em programas multidisciplinares preventivos, antes de uma futura gravidez. Quando houver possibilidade de retardar a concepção, a mulher obesa deve seguir programas para adequação de peso, e, se possível, atingir IMC normal, para que possam engravidar e tenham menores riscos a si, e aos seus recém-nascidos. ${ }^{29,30}$

\section{CONCLUSÕES}

1. Recém-nascidos de uma amostra de mães obesas teve maiores peso ao nascer, perímetro braquial, perímetro cefálico e comprimento do que os de pacientes com IMC normal.

2. Os parâmetros antropométricos e características volumétricas dos recém- nascidos de mães obesas, podem ter contribuído para maior taxa de partos cirúrgicos, quando comparados aos do grupo de pacientes com IMC normal.

3. Mulheres obesas em idade reprodutiva, devem ser informadas de seus riscos associados antes de uma futura gestação. 


\section{REFERÊNCIAS}

1. Ober C, Steck T, van der Ven K, Billstrand C, Messer L, Kwak J, Beaman K, Beer A. MHC class II compability in aborted fetuses and term infants of couples with recurrent spontaneous abortion. J Reprod Immunol. 1993; 25 (3): 195-207.

2. Poole JA, Claman HN. Immunology of pregnancy. Clin Rev Allergy Immunol. 2004; 26 (3): 16170.

3. Hunt JS. Stranger in a stranger land. Immunol Rev 2006; 231: 36-47. DOI: 10.1111/j.1600065X.2006. 00436.x

4. Cabar FR, Codarin RR, Bunduki V. In: Zugaib M, editor. Obstetrícia. Placenta, sistema amniótico e cordão umbilical. $3^{\mathrm{a}}$ ed. São Paulo: Manole, 2016. p. 66-87.

5. Brock RS, Falcão MC. Avaliação nutricional do recém-nascido: limitações dos métodos atuais e novas perspectivas. Rev Paul Pediatr. 2008; 26 (1): 70-6.

6. Rudge MCV, Borges VTM, Calderon IMP. In: Neme B, editor. Obstetrícia Básica. Adaptação do Organismo Materno à Gravidez. $3^{\mathrm{a}}$ ed. São Paulo: Sarvier; 2006. p. 36-62..

7. Magana CB, Zugaib M. In: Neme B, editor. Obstetrícia Básica. Obesidade. $3^{\mathrm{a}}$ ed. São Paulo: Sarvier; 2006. p. 1316-8..

8. Abreu IF, Silva AF, Côrtes ALS, Silva FQN, Albuquerque JPM, Amaral Neto JC, Silva NRO, Gil FR. Associação entre gestação e síndrome metabólica: Repercussões materno-fetais e desafios diagnósticos. Braz J Develop. 2021; 7 (12): 110447-64.

9. Modder J, Fitzsimons KJ. Centre for Maternal and Child Enquiries. Royal College of Obstetricians and Gynaecologists. CMACE/RCOG Joint Guideline Management of Women with Obesity in Pregancy. 2010. DOI 10.1385/CRIA: 26: 3: 161

10. Mattar R, Torloni MR, Betrán AP, Merialdi M. Obesidade e gravidez. Rev Bras Ginecol Obstet. 2009; 31 (3): 107-10.

11. Linn CC, Mahmood T. Obesity in pregnancy. Best Pract Res Clin Obstet Gynaecol. 2015: 29 (3): 309-19.

12. Asplund CA, Seehusen DA, Callahan TL, Olsen C. Percentage Chance in Antenatal Body Mass Index as a Predictor of Neonatal Macrosomia. Ann Fam Med. 2008; 6 (6): 550-4.

13. Institute of Medicine. Subcommittee on Nutritional Status and Weight Gain During Pregnancy. Nutrition during pregnancy: Weight gain and nutrient supplements. National Academy Press. Washington, DC. 1990; Part I: 27-233. DOI: 10.17226/1451

14. Yoshizack CT, Fittipaldi FS, Osmundo Jr GS, Francisco RPV, Martinelli S, Bunduki V. In: Zugaib M, editor. Obstetrícia. Assistência ao trabalho de parto. $3^{a}$ ed. São Paulo: Manole, 2016. p. 370-85.

15. Battaglia FC, Lubchenko LO. A practical classification of newborn infants by weight and gestational age. J Pediatr. 1967; 71 (2): 159-63. 
16. Cardoso LE, Falcão MC. Importância da avaliação nutricional de recém-nascidos pré-termo por meio das relações antropométricas. Rev Paul Pediatr. 2007; 25 (2):135-41.

17. Figueira, BBD; Segre, CAM. Mid-arm circumference and mid-arm/head circumference ratio in term newborns. São Paulo Med J. 2004; 122 (2):53-9.

18. Capurro H, Konichezky S, Fonseca D, Caldeyro-Barcia R. A simplified method for diagnosis of gestational age in the newborn infant. J Pediatr. 1978; 93 (1): 120-2.

19. Apgar V. A proposal for a new method of evaluation of newborn infant. Rev Anesth Analg. 1953; 32 (4): 260-7.

20. Código de Nuremberg e Declaração de Helsinki; [acesso em 04 dez 2021]. Disponível em: http://www.efdeportes.com/efd183/codigo-de-nuremberg-e-declaracao-de-helsinki.htm.

21. Resolução 466/12- Conselho Nacional de Saúde; [acesso em 04 dez 2021]. Disponível em: http://conselho.saude.gov.br/resolucoes/2012/Reso466.pdf

22. Junqueira SM, Tsunechiro. A parturiente no contexto da sala de parto: necessidades básicas afetadas. Rev Esc Enf USP. 1988; 22: 148-60.

23. Siegel S, Castelani Jr NJ. Estatística não-Paramétrica Para Ciências do Comportamento. $2^{\mathrm{a}}$ ed. Porto Alegre: Artmed. 2006. p. 448.

24. Landis JR, Kock GG. The measurement of observer agreement for contrasts among multinomial populations. Biometrics. 1977; 33 (1): 159-74.

25. Rosner B. Fundamentals of Biostatistics. $7^{\text {th }}$ ed. 2011.Cengage Learning Customer $\&$ Sales Support, 1-800-354-9706.

26. Pires IG, Gonçalves DR. Consumo alimentar e ganho de peso de gestantes assistidas em Unidade Básica de Saúde. Bras J Health Rev. 2021; 4 (1): 128-46.

27. Kashan AS, Kenny LC. The effects of maternal body mass índex on pregnancy outcome. Eur J Epidemiol. 2009; 24 (11): 697-705.

28. Padilha PC, Saunders C, Machado RCM, Silva CL, Bull A, Sally EOF, Acciolly E. Associação entre o estado nutricional pré-gestacional e a predisposição do risco de intercorrências gestacionais. Rev Bras Ginecol Obstet. 2007; 29 (10): 511-8.

29. Novo JLVG, Oliveira AAA, Baylão LB, Fenili P, Novo NF. Impactos das assistências obstétricas e dos tipos de partos: experiências de primíparas. Lat Amer J Develop. 2021; 3 (5): 3145-57.

30. González-Plaza E, Bellart J, Martinez-Verdú MÁ, Arranz Á, Barroso LL, Seguranyes G. Prepregnancy overweight and obesity prevalence and relation to maternal and perinatal outcomes. Enferm Clin. 2021. Jun 19: S1130-8621(21)00081-4. 